\title{
Highly Anisotropic Rigidity of "Ribbon-Like" Polymers: II. Nematic Phases in Systems between Two and Three Dimensions
}

\author{
Irina A. Nyrkova $\left({ }^{1,2}\right)$, Alexander N. Semenov $\left({ }^{1,2,3,4, *}\right)$ and \\ Jean-François Joanny $\left({ }^{2}\right)$ \\ ( ${ }^{1}$ Physics Department, Moscow State University, 117234 Moscow, Russia \\ $\left({ }^{2}\right)$ Institut Charles Sadron, 6 rue Boussingault, 67083 Strasbourg Cedex, France \\ $\left({ }^{3}\right)$ Department of Applied Mathematics, University of Leeds, Leeds LS2 9JT, UK \\ ( ${ }^{4}$ ) Nesmeyanov Institute of Organo-Element Compounds of Russian Academy of Science, \\ 28 Vavilova Str., 117812 Moscow, Russia
}

(Received 25 October 1996, received in final form 26 February 1997, accepted 28 February 1997)

PACS.61.25.Hq - Macromolecular and polymer solutions, polymer melts; swelling

PACS.61.30.-v - Liquid crystals

PACS.64.70.-p - Specific phase transitions

\begin{abstract}
Various extensions of Onsager theory based on the second virial approximation are constructed in order to describe nematic P-phases in ribbon polymer solutions. If the average coil shape is anisotropic and the concentration is not too high, the coils can be considered as anisotropic solid objects and an ordinary expansion over the coil concentration can be performed. This first approach can be applied to study pancake nematic ordering. At higher concentrations and when the polymer chains are rather long, the polymer solution can be considered as a solution of short fragments connected into chains with a particular statistics reflecting the polymer structure with two rigidities; a similar virial expansion can be constructed. This last approach allows the consideration of the various symmetries of the nematic phases simultaneously. We also take into account the correlation correction to the mean field result which provides an essential additional angular dependence to the interaction free energy. Scaling arguments are applied in the case where the intra-coil correlations are very strong, or if the coils interact as opaque objects. The methods developed here will be used in further publications to study liquid crystalline ordering in solutions of ribbon chains.
\end{abstract}

\section{Introduction}

A new family of semirigid polymers, ribbon-like ladder polymers has become available quite recently $[1,2]$ (Fig. 1a). A similar tape-like structure is also characteristic of some living polymers formed by rod-like peptides [3] (Fig. 1b). These ribbon polymers possess an unusual rigidity: they rather freely bend inside one plane (the plane of main flexibility, PMF), but their out of plane rigidity is extremely high. We have recently considered in the first paper of this series the behavior of isolated polymer chains "with planar rigidity" (or "with two-dimensional

${ }^{*}$ ) Author for correspondence (e-mail: A.Semenov@leeds.ac.uk) 

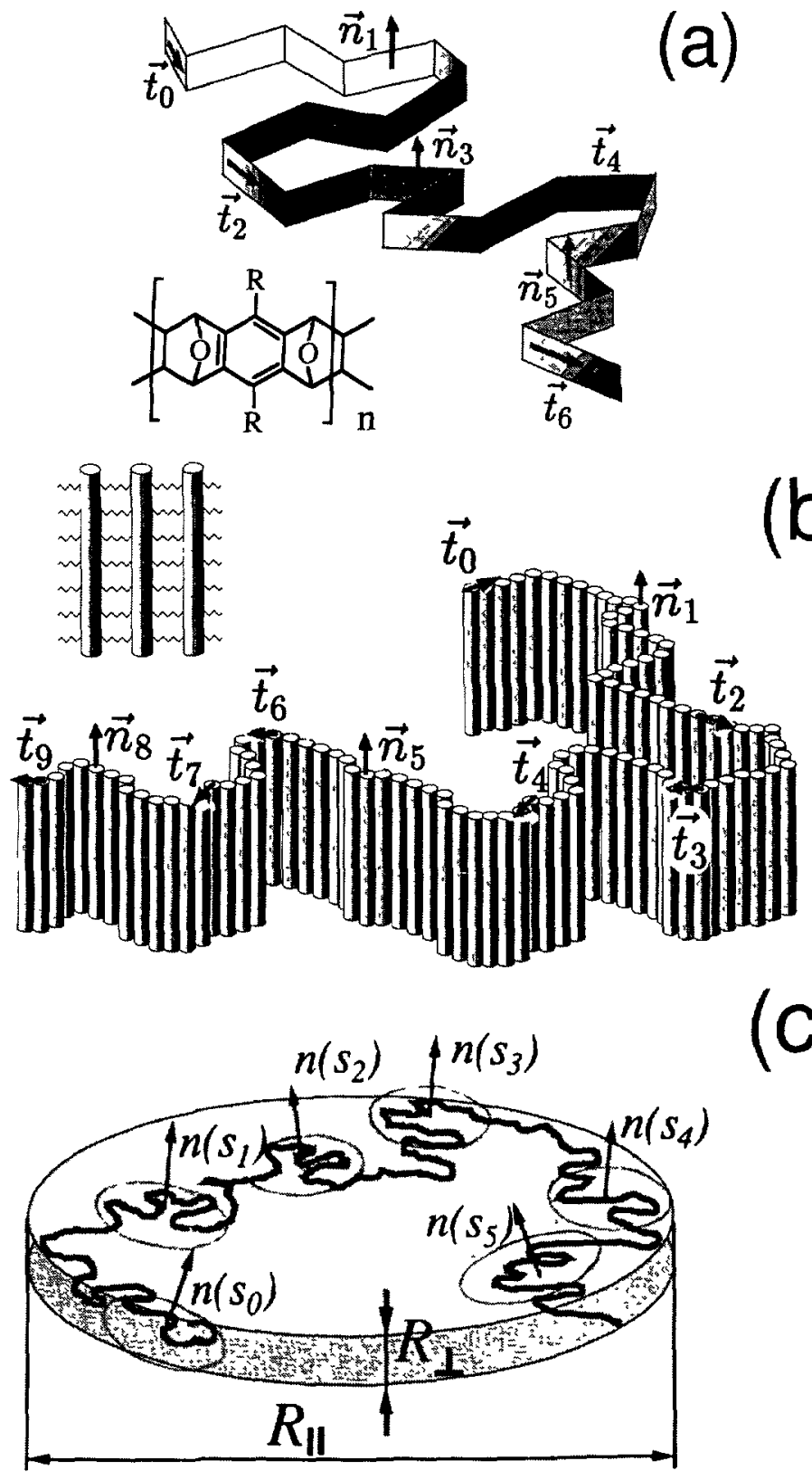

(c)

Fig. 1. - Ribbon-like polymers with anisotropic rigidity. (a) Soluble ladder macromolecular chain with mirror symmetry and its local conformation. (b) Living tape-like polymer formed by peptide rodlike fragments and its local structure (the rods attract each other by the opposite sides). (c) Typical conformation of the polymer with two-dimensional flexibility (short fragments are crumbly discs with the radius $R_{\|}$and the thickness $R_{\perp} \ll R_{\|}$). The local orientation of a ribbon chain can be characterized by the tangent vector $\mathbf{t}$ and by the vector $\mathbf{n}$ normal to the plane of main flexibility (PMF). 

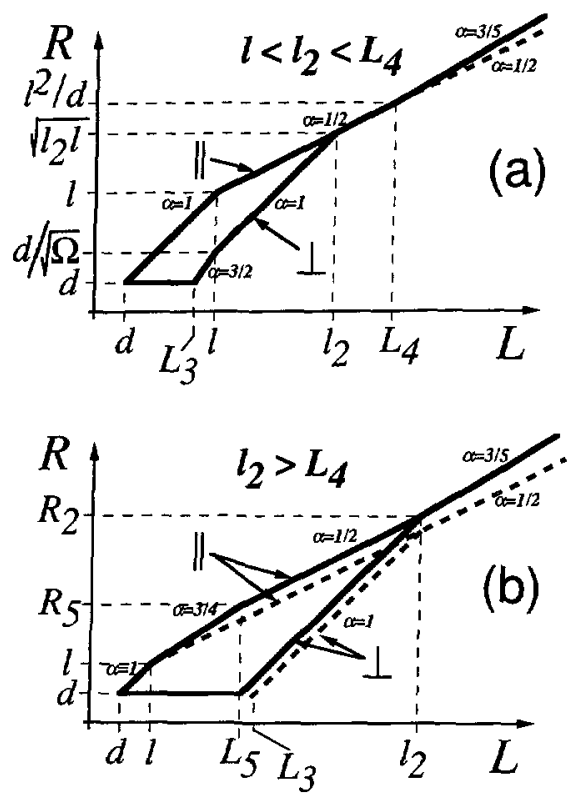

Fig. 2. - Characteristic scales $R_{\|}$and $R_{\perp}$ plotted vs. contour length $L$ of a chain fragment for various values of the second rigidity $l_{2}$ : (a) $(d / l)^{2} \lesssim \Omega \lesssim 1$; (b) $\Omega \gtrsim 1$. $L_{4} \equiv l^{3} / d^{2}$. The slopes $\alpha$ for the dependencies $R(L) \propto L^{\alpha}$ are shown near the corresponding lines. Self-avoiding chain (solid lines); ideal chain (dashed lines).

flexibility", if one prefers). In the following, we will refer to this work as paper I [4] (the references to the sections and equations of this paper are written below as "I.x.y" where "x.y" is the number from Paper I). We have shown that the anisotropy of the rigidity induces a shape anisotropy of the chains, which tend to adopt a disc-like conformation (Fig. 1c). The shape anisotropy in turn can cause an orientational ordering of the coils and in particular lead to the formation of nematic phases with various symmetries (which we named $\mathrm{P}-, \mathrm{N}-$ and B-nematics).

The local orientation of a ribbon chain can be characterized by the tangent vector $\mathbf{t}$ and by the vector $\mathbf{n}$ normal to the PMF (Fig. 1). In paper I, we have justified persistent-like models for the chain statistics of ribbon polymers (with two persistence lengths $l$ and $l_{2}$ ); the orientation correlations of vectors $\mathbf{t}$ and $\mathbf{n}$ are decaying exponentially:

$$
\langle\mathbf{t}(0) \mathbf{t}(s)\rangle=\exp (-s / l), \quad\langle\mathbf{n}(0) \mathbf{n}(s)\rangle=\exp \left(-s / l_{2}\right), \quad l_{2} \gg l,
$$

where $s$ is the linear coordinate along the chain contour. The chain conformation essentially depends on the dimensionless parameter

$$
\Omega \equiv \frac{l_{2} d^{2}}{l^{3}}
$$

where $d$ is the chain thickness (see Fig. 2). At large values of $\Omega(\Omega \gtrsim 1)$ relatively short chain fragments (with contour lengths $s: l \lesssim s \lesssim L_{5} \equiv l \Omega^{2 / 5}$ ) look like absolutely flat coins (the perpendicular size is $R_{\perp} \simeq d$ ), at larger scales $\left(L_{5} \lesssim s \lesssim l_{2}\right)$ the chain adopts a thick disc conformation (where $R_{\perp} \gtrsim d$, but the coils are still anisotropic: $R_{\|} \gg R_{\perp}$ ) (Fig. 2b). For smaller values of $\Omega\left(d^{2} / l^{2} \lesssim \Omega \lesssim 1\right.$, or $\left.l \lesssim l_{2} \lesssim L_{4} \equiv l^{3} / d^{2}\right)$ the flat coin regime disappears, however the conformation is still anisotropic (disc-like) for $l \lesssim s \lesssim l_{2}$ (Fig. 2a). 
It is well-known that lyotropic nematic crystals are formed in rather concentrated solutions of semirigid polymers. Ordinary semiflexible chains order in a nematic phase $(N-$ phase) where the main axis of the chains (the tangent vectors $\mathbf{t}$ ) lay nearly parallel to each other [5-7]. Disc-like objects tend to order in another type of liquid crystalline phase where the disc planes (the PMFs in the case of ribbon polymers) tend to have a parallel orientation $[5,8-10]$, we called this phase a pancake nematic $(P-$ nematic). A biaxial nematic $(B-$ nematic) phase is formed when both $\mathbf{t}$-vectors and $\mathbf{n}$-vectors are ordered (obviously in perpendicular directions). For more detailed explanations about the phases $\mathrm{P}, \mathrm{N}$ and $\mathrm{B}$ we refer readers to the paper $\mathrm{I}$.

It is natural to use the Onsager approach [11] to investigate these lyotropic nematic phases. In particular, the classical Onsager second virial approximation is asymptotically exact for the description of the isotropic-nematic transition in solutions of rods (or ordinary semiflexible chains) in the limit of large shape anisotropy (when the axial ratio $d / l \rightarrow 0$ ): the corrections due to higher terms in the virial expansion are negligible in this limit [12]. In other words, the correlation corrections to the mean field treatment (the second virial approximation) are small when $d / l \rightarrow 0$. Note however, that this statement is true in the three-dimensional case only: for two-dimensional solutions of rods the correlation corrections are quite significant [12].

Polymers with asymmetric rigidity show an intermediate behavior between the three- and two-dimensional limits. Indeed, the solution itself is three-dimensional. However, the chains are disc-like, and for high enough second rigidity $l_{2}$, they have a planar ( $f l a t$ ) conformation at small contour length scales. The coils themselves are close to two-dimensional objects for which the correlation corrections are essential. The relative importance of the intra-chain interactions (with strong correlations) vs. interchain interactions, must be analyzed carefully in each specific case.

The aim of the present paper is to study the validity of the classical Onsager approach in the case of ribbon-like polymers. In this paper the discussion is limited to ordering of P-type only, and our main task is to calculate the free energy of the ordered P-phase. Ordering of the B-type can be studied in a similar manner, and we postpone its consideration to the next paper.

The following two types of Onsager-like theories are known $[6,7]$ :

(1) If the objects in solutions have a shape anisotropy, one can characterize the nematic phase by an orientational distribution function of the objects, as if they were solid. The second virial approximation is accurate for solutions of anisotropic objects provided that the concentration is low enough. For ordinary solid opaque discs (of diameter $R_{\|}$) the second virial approximation breaks down at concentrations of order $R_{\|}^{-3}$, i.e. in the range of the orientational transition concentration $[5,12]$. However, the discs formed by ribbon polymers are "crumbly" (loose): the trajectory of the chain occupies only a small fraction of the disc volume. Hence, the region of validity of the second virial approximation can be wider. The generalization of this kind of Onsager theory (which we will further refer to as global approach) to the case of ribbon-like polymers is discussed below in Section 2 .

(2) For semirigid polymers, at a higher concentration (when the coils overlap strongly) a similar theory based on the second virial approximation can be constructed, but as an expansion over the segment concentration rather than of the chain concentration. The corresponding approach will be referred to in the following as local. The intrinsic conformational entropy of the chains is taken directly into account in this approach which thus treats rigorously the effect of chain flexibility. This approach has been used successfully for solutions of ordinary semiflexible chains [13]. We reformulate it for the particular case of chains with anisotropic rigidity below in Section 3 . 
In Section 4, we consider the role of correlation corrections to the second virial approximations for ribbon-like polymers. We calculate the first order correlation corrections to the second virial coefficients.

The following notations are used: $\Phi$ is the volume fraction occupied by the polymer chains in solution, $\Phi \sim l d^{2} n \sim L d^{2} c$, where $n$ is the concentration of $l$-segments and $c$ is the chain concentration; $L=N l$ is the contour length of a chain. For a formal analysis of the ordered phases, one needs a probe function to characterize the orientational distribution functions, we use here the distribution introduced in paper I (Eq. (I.4.1)). Accordingly, the order parameters in the nematic phases are characterized by orientation angles $\gamma$ (for the director $\mathbf{t}(s)$ ) and $\psi_{1}$, $\psi_{2}$ (for the director $\mathbf{n}(s)$ ). The isotropic state corresponds to $\Gamma \equiv \sin \gamma=1, \Psi_{1} \equiv \sin \psi_{1}=1$, $\Psi_{2} \equiv \sin \psi_{2}=1$. The pancake (P) nematic corresponds to $\Gamma=1, \Psi_{1}=\Psi_{2}=\Psi<1$, and the ordinary nematic (N) state is characterized by $\Psi_{1}=1, \Gamma=\Psi_{2}<1$. As in the previous paper, we use energetic units $\left(k_{\mathrm{B}} T=1\right)$. The dimensions of a disc-shaped coil are denoted as $R_{\|}$(radius) and $R_{\perp}$ (width) (Fig. 1c). The following contour lengths are also relevant $L_{3} \equiv l \max \left\{\Omega^{1 / 3}, \Omega^{1 / 2}\right\}, L_{4} \equiv l^{3} / d^{2}, L_{5} \equiv l \Omega^{2 / 5}, R_{5} \equiv l \Omega^{3 / 10}$

\section{Nematic Ordering in Solutions of Soft Crumbly Discs: Low Concentration Regime}

In this section we describe the general idea of the Onsager-like theories for solutions of soft crumbly (loose) disc-like objects formed by polymers with anisotropic rigidity, assuming that the polymer volume fraction is low enough. Here we adopt a global approach assuming that the elementary unit of the system is a disc-like object (one chain).

The free energy of a solution of anisotropic objects can be written as a sum of three terms [14]:

$$
\mathcal{F}=\mathcal{F}_{\text {gas }}+\mathcal{F}_{\text {ord }}+\mathcal{F}_{\text {int }}
$$

where $\mathcal{F}_{\text {gas }}$ is the "ideal-gas" free energy corresponding to the translational entropy of the objects, $\mathcal{F}_{\text {ord }}$ corresponds to the entropy loss due to the ordering, and $\mathcal{F}_{\text {int }}$ is the energy of interaction between the objects.

The interaction term, $\mathcal{F}_{\text {int }}$, can be calculated within the second virial approximation if the concentration is not too high (the precise condition is formulated in Appendix $C$, Eqs. (C.5, C.6)). For disc-like objects the free energy (2.1) per object is:

$$
\begin{aligned}
\mathcal{F}_{\text {gas }} & \simeq \ln c \\
\mathcal{F}_{\text {ord }} & \simeq \int f \ln (4 \pi f) \mathrm{d}^{2} \Omega_{\mathbf{n}} \sim-\ln \Psi \\
\mathcal{F}_{\text {int }} & \simeq \frac{c}{2} \iint f(\mathbf{n}) f\left(\mathbf{n}^{\prime}\right) B\left(\mathbf{n}, \mathbf{n}^{\prime}\right) \mathrm{d}^{2} \Omega_{\mathbf{n}} \mathrm{d}^{2} \Omega_{\mathbf{n}^{\prime}} \sim c B(\psi)
\end{aligned}
$$

where $c$ is the number of discs per unit volume, $f(\mathbf{n})$ is the orientation distribution function of the normal vectors $\mathbf{n}$ which determine the disc orientations, and $B(\psi)$ is the second virial coefficient between two objects with a relative angle $\psi$ between their axes.

The free energy, given in equations $(2.1,2.2)$ must then be minimized with respect to the orientational distribution function, $f(\mathbf{n})$. The minima correspond to the possible liquid crystalline phases (isotropic, P-nematic, etc.); the transitions between these phases are finally studied in a standard way by equating both the polymer chemical potentials and the osmotic pressures in the coexisting phases.

For ordinary solid discs:

$$
B(\psi) \sim R_{\|}^{3} \sin \psi
$$


The free energy $(2.1,2.2)$ implies an orientational transition from the isotropic phase (I-phase) to the pancake nematic (P-phase) at

$$
c_{\mathrm{I}} \sim c_{\mathrm{P}} \sim\left(c_{\mathrm{P}}-c_{\mathrm{I}}\right) \sim 1 / R_{\|}^{3} .
$$

The characteristic orientation angle in the P-phase is

$$
\psi \sim\left(c R_{\|}^{3}\right)^{-1}
$$

Although the second virial approximation is not exact for the transition point, one can use the results $(2.4,2.5)$ as scaling estimates (cf. [5]).

As already mentioned above, the discs made of ribbon-like chains with two rigidities are actually very crumbly (loose), so that the results known for solid discs cannot be applied directly. However, the general approach (Eqs. $(2.1,2.2)$ ) is still useful provided that one recalculates the second virial coefficient $B(\psi)$ between the crumbly discs.

The second virial coefficient between two disc-shaped coils with orientations $\mathbf{n}$ and $\mathbf{n}^{\prime}$, is calculated from the general relations (see Ref. [11]):

$$
B\left(\mathbf{n}, \mathbf{n}^{\prime}\right) \equiv \int \beta\left(\mathbf{r}, \mathbf{n}, \mathbf{n}^{\prime}\right) \mathrm{d} \mathbf{r}
$$

with

$$
\beta\left(\mathbf{r}, \mathbf{n}, \mathbf{n}^{\prime}\right) \equiv\left[1-\exp \left(-U\left(\mathbf{r}, \mathbf{n}, \mathbf{n}^{\prime}\right)\right)\right] \simeq\left\{\begin{array}{cll}
U-U^{2} / 2, & \text { if } U \lesssim 1 ; & \text { (a) } \\
1, & \text { if } U \gtrsim 1 . & \text { (b) }
\end{array}\right.
$$

where $U\left(\mathbf{r}, \mathbf{n}, \mathbf{n}^{\prime}\right)$ is the effective interaction free energy between the two discs with their centers of mass separated by vector $\mathbf{r}$. Line (a) in equation (2.6) corresponds to the regime of transparent discs; line (b) reflects the hard core excluded volume approximation. For solid discs ( $U=\infty$ provided that the discs do intersect): we obtain the well-known result (2.3) using line (b) of equation (2.6). For crumbly discs we need first to calculate the interaction energy $U$.

The interaction energy $U$ can be estimated using scaling arguments (see Appendix A) in the regime $\Omega \gtrsim 1$, where the structure of the chain is essentially two-dimensional on large enough contour length scales (up to $s \sim L_{5}, L_{5} \simeq l \Omega^{2 / 5} \gg l$ ); the result is given by equations (A.2, A.4). For lower second rigidities $(\Omega \lesssim 1$ ), or for large enough angle $\psi$ between the disc orientations $\left(\Omega \gtrsim 1\right.$, but $\psi \gtrsim \psi_{\mathrm{mf}} \simeq d / l$, (A.5)) the energy $U$ can be calculated via a mean field approximation (see Appendix B); the results are summarized in equation (B.3, B.5).

For $\Omega \gtrsim 1$ at very low angle $\psi$, the coils interact strongly (with $U \gtrsim 1$ ), and the second line of equation (2.6) leads to a virial coefficient $B(\psi)$ identical to that between opaque discs. In the scaling regimes $(\Omega \gtrsim 1$ and $\psi \lesssim d / l)$ and for larger angle, when the interaction is weak, we use the approximation $\beta \simeq U$, line (a) of equation (2.6):

$$
B(\psi) \sim U R_{\|}^{3} \Psi \sim L^{2}\left[l^{2} d \Psi^{2}\right]^{1 / 3}
$$

where $\Psi=\sin \psi$. Both equations (A.2) and (A.4) lead to the same value of the virial coefficient. It is clear that the second virial coefficient given by equation (2.7) favors the formation of a pancake nematic P-phase. However in the "mean field regimes" $\psi \gtrsim d / l$ the second lines of equations (A.2, A.4) give $B(\psi) \sim L^{2} d$, that is no angular dependence. As discussed in Appendix $\mathrm{B}$, this is because we omit numerical prefactors within the scaling approximation used to calculate the energy $U$. A more detailed consideration given in the Appendix B leads to a more accurate result (Eq. (B.4)):

$$
B(\psi) \sim L^{2} d b(\Psi), \quad \text { where } \quad b(\Psi) \simeq 1+\Psi^{2}
$$


(see Eq. (B.3); the numerical constant in this equation is set to 1 as its particular value is irrelevant for our scaling analysis) which reaches a constant value both at small and at large values of the angle. Again a pancake nematic ordering is favored since $b(\Psi)$ is an increasing function of $\Psi$.

Thus the formation of ordered phases in all the cases is driven by the interaction free energy. For the transition point (when $\Psi \sim 1$ ) the second virial coefficient is given by equation $(2.3$ ) for "opaque discs" and by equation (2.8) for "semi-transparent discs" (when $U \lesssim 1$ ).

The analysis of the free energy of crumbly anisotropic discs solution (Eqs. $(2.1,2.2)$ ) within the mean field approximation (see Eq. (2.8) or Eqs. (B.4)) shows that a first order transition I-phase $\rightarrow$ P-phase takes place at the concentrations

$$
c_{\mathrm{I}} \sim c_{\mathrm{P}} \sim\left(c_{\mathrm{P}}-c_{\mathrm{I}}\right) \sim\left(L^{2} d\right)^{-1}
$$

Above the transition point the characteristic orientation angle in the $\mathrm{P}$-phase is

$$
\psi \sim\left(c L^{2} d\right)^{-1 / 2}
$$

The results $(2.9,2.10)$ are valid if $U \lesssim 1$ and $\psi>d / l$. Near the transition point, the condition $U \lesssim 1$ is equivalent to $L^{2} d R_{\|}^{-3} \lesssim 1$. In the limit $\Omega \lesssim 1$, it is satisfied up to $L \lesssim L_{4}$, i.e. in the whole region of anisotropic discs (since $L_{4} \gg l_{2}$ ). In the limit $\Omega \gtrsim 1$, the condition $L^{2} d R_{\|}^{-3} \lesssim 1$ is equivalent to $L \lesssim L_{6}$, where

$$
L_{6} \equiv l_{2} \Omega^{-2 / 5} \equiv\left(l^{3} / d^{2}\right) \Omega^{3 / 5}
$$

If $L \gtrsim L_{6}$, then $U \gtrsim 1$, so that the second virial coefficient is given by equation (2.3). In this regime the chains behave like opaque discs.

As can be seen from Appendix C (condition (C.5)), the higher order terms in the virial expansion have no influence on the result (2.9) for the regime of transparent discs (as $R_{\|}^{3} \gg L^{2} d$, if $U \ll 1$ ). For the opaque discs regime (condition (C.6)) the higher order terms are important at the transition point, however these terms change only the numerical prefactors and do not lead to a qualitatively different behavior.

We also show below that the correlation corrections to the mean field approximation are unimportant for $\Psi \sim 1$ (see Sect. 4, Eqs. $(4.15,4.17)$ ). Thus the $I \rightarrow P$ transition concentration is well-described by equation (2.9) for all the regimes of anisotropic discs $\left(L \lesssim l_{2}\right)$ provided that either $\Omega \lesssim 1$, or $\Omega \gtrsim 1$ and $L \lesssim L_{6}$. For $\Omega \gtrsim 1$ and $L_{6} \lesssim L \lesssim l_{2}$ the transition is described by equation $(2.4)$.

\section{Liquid Crystalline Ordering of Ribbon-Like Polymers: Strongly Overlapping Coils}

The Onsager approach, based on the global second virial coefficient between two coils (see Eqs. $(2.1,2.2)$ ), is suitable only when the coils can be considered as solid-like anisotropic objects i.e. at low enough concentrations (see Appendix C). This approach is not applicable if the average coil shape is isotropic (when the polymers are long enough: $L \gtrsim l_{2}$ ), and if the coils strongly overlap. However, in this case the anisotropic internal coil statistics can still lead to a nematic ordering.

In a semidilute solution where the polymer coils overlap strongly, we study the ordering transition along the lines $[13,14]$ first proposed by one of us for semiflexible chains with isotropic rigidity. This approach is also based on the Onsager second virial approximation, but it is short 
chain fragments (rather than whole chains) that are now considered as the elementary units. We reformulate here this approach (which we call the local approach) for the particular case of chains with two rigidities.

Let us divide the chains into short segments of contour length $\lambda$, chosen so that $d \ll \lambda \ll l \lesssim l_{2}$, and consider the solution of long chains as a solution of such segments (of length $\lambda$ and diameter $d$ ). The concentration of segments is $\nu \equiv c L / \lambda$, where $c$ is the chain concentration. The segments are not completely free, but are connected to each other (into semiflexible chains), and their positions and orientations are correlated. For chains with two rigidities, two vectors, tangential, $\mathbf{t}(s)$ and normal, $\mathbf{n}(s)$, characterize the segment orientation; the local correlations between these vectors are defined by equation (1.1).

The free energy of the system in the $P$-phase (with an order parameter $\Psi$ ) is written as in equation (2.1). The term $\mathcal{F}_{\text {gas }}$ is the translational entropy of the chains, i.e. the ideal gas entropy (per chain):

$$
\mathcal{F}_{\text {gas }}=\ln c \text {. }
$$

To estimate the entropy loss due to an orientational ordering we use the following scaling procedure. The first segment of each chain should be oriented properly; this costs an entropy $\Delta S \sim \ln (\Psi)$. After that the chain spreads in space freely, segment after segment, according to the equilibrium statistics, until the angle between the vector $\mathbf{n}(s)$ and the corresponding axis becomes greater than $\psi$. At this point we "correct" the orientation of the segment, and each "correction" costs one degree of freedom or an entropy $\Delta S \sim 1$. The deflection length between the correction points, $\lambda_{\psi}$, is:

$$
\lambda_{\psi} \sim l_{2} \Psi^{2}
$$

Thus, the free energy connected with the orientation of the segments $(\lambda)$ is

$$
\mathcal{F}_{\text {ord }} \simeq \ln \left(\frac{1}{\Psi}\right)+\frac{L}{\lambda} \frac{\lambda}{l_{2}} \Psi^{-2}
$$

(for $\Psi \ll 1$ ). The numerical coefficients are omitted in equation (3.3) and below. A more rigorous quantitative derivation of the analog of equation (3.3) including numerical coefficients obtained following the lines of references $[7,13,14]$ confirms the scaling result.

The final term $\mathcal{F}_{\text {int }}$ in the free energy is due to the interaction between the segments. Since the segments are rod-like $(\lambda \gg d)$, we can use the second virial approximation to estimate $\mathcal{F}_{\text {int }}$ provided that the volume fraction occupied by the chains, $\Phi \sim \nu d^{2} \lambda$, is small, $\Phi \ll 1$ (cf. Eqs. (B.2, 2.2))

$$
\mathcal{F}_{\mathrm{int}} \equiv \frac{L}{\lambda} \frac{\nu}{2} \iint f(\mathbf{t}) f\left(\mathbf{t}^{\prime}\right) B_{\lambda}\left(\mathbf{t}, \mathbf{t}^{\prime}\right) \mathrm{d} \Omega_{\mathrm{t}} \mathrm{d} \Omega_{\mathbf{t}^{\prime}} \sim \frac{L}{\lambda} \nu d \lambda^{2} b(\Psi),
$$

where $B_{\lambda}\left(\mathbf{t}, \mathbf{t}^{\prime}\right)=2 \lambda^{2} d \sin \left(\mathbf{t}, \mathbf{t}^{\prime}\right)$ is the second virial coefficient of two segments, and the function $b(\Psi)$ is defined in equation $(2.8)$.

Combining equations (3.1-3.4) we get the following formula for a free energy (per chain) of a solution of chains with two rigidities, $l$ and $l_{2}$ :

$$
\mathcal{F} \sim \ln \Phi+\ln \left(\frac{1}{\Psi}\right)+\frac{L}{l_{2}} \Psi^{-2}+\Phi \frac{L}{d} b(\Psi)
$$

where $\Phi$ is the volume fraction occupied by the chains (see notation (2.8)). Note that the result, equation (3.5) is approximate: it is asymptotically valid for both strongly anisotropic $(\Psi \ll 1)$ and for nearly isotropic $(\Psi \simeq 1)$ states. Thus this equation is appropriate for our scaling analysis of the phase behavior. 
In principle, a complete analysis of the behavior of solutions of chains with two rigidities is possible on the basis of equation (3.5): at any given $\Phi$ one can minimize the free energy (3.5) with respect to the parameter $\Psi=\sin \psi$, and then get the phase diagram (for the $\mathrm{I}$ and $\mathrm{P}$ phases) using the standard techniques (see, e.g. Refs. $[11,14]$ ).

It is important to note that the analysis presented here (Eqs. $(3.3,3.4)$ ) is based on the mean field approximation. In particular the derivation of equation (3.3) is based on the assumption that the orientation correlations are described by equations (1.1) and are not essentially disturbed by the excluded volume interactions. The virial coefficient in equation (3.4) is local: it accounts for the direct interaction between segments. The effect of the chain structure is neglected, i.e. it is assumed that the correlations between the positions of the chain segments are effectively screened. In the case where the correlations are strong enough (as we will see below in Sect. 4, this is a rather common situation for solutions of chains with planar rigidity), the corresponding corrections must be added to the free energy functional (3.5).

\section{Correlation Corrections to the Free Energy of Semidilute Solutions of Chains with Planar Rigidity}

It has been shown by Edwards [15] that the mean field approximation for semiflexible chains, such as the one used above, is valid when the excluded volume interactions are screened. For solutions of ordinary semiflexible chains $\left(l_{2}=l\right)$ this requires that the volume fraction $\Phi$ occupied by the chains be higher than $d^{3} / l^{3}$, or in other words, that the concentration blobs are not swollen. The contour length of the blob in $3 \mathrm{~d}$-space is $\lambda \sim d^{4} /\left(l^{3} \Phi^{2}\right)$; thus the blobs are not swollen if $\lambda \lesssim l^{3} / d^{2}$. That means that the chains must overlap strongly at the scales where the total interaction energy is of order $k_{\mathrm{B}} T$.

In the case of chains with two-dimensional flexibility we must distinguish interactions between segments of the same chain and between segments of different chains even in the case where the chains strongly overlap. Indeed, locally each chain is a two-dimensional coil, hence the intra-chain correlations can be very pronounced (note that two dimensional chains are always swollen if $L>l$ ). In the limit $l_{2} \rightarrow \infty$ the chain retains the initial PMF orientation forever: the probability of a binary collision with a segment of the same chain (which is controlled by the two-dimensional concentration in the PMF) can be much higher than the probability of a collision with a segment of another chain (which depends on the overall three-dimensional concentration) even well inside the semidilute regime when the coils overlap strongly.

Our aim now is to study correlations in solutions of flat (or semi-flat) coils and to calculate the corresponding corrections to the free energies beyond the mean field approximations given by equation (3.5) (local approach) (or by Eqs. (2.1, 2.2, B.4) (global approach)). As is shown in Appendix $\mathrm{C}$, for the case of transparent disc-like chains the second virial approximation is valid (the higher order virial terms constitute only a small correction). However, there is a problem connected with calculation of the second virial coefficient itself, as we should use the virial coefficient between fragments connected into chains, rather than the virial coefficient between free cylindrical fragments, used in equation (3.4). Below we estimate the corrections to the second virial coefficient related to the fact that the fragments are connected and form a long flat (or semi-flat) chain (see Eqs. $(4.15,4.17)$ ). We use a mean field technique in the calculations below and find the first order corrections to the pure mean field results for the second virial coefficient.

4.1. General Result for the Correlation Correction Term. - The interaction contribution to the free energy (per unit volume) of the solution can be written in the following 


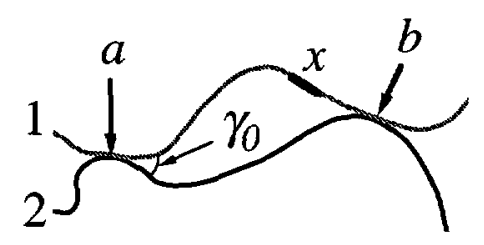

Fig. 3. - Two semiflexible polymer chains intersecting twice, at points $a$ and $b ; \gamma_{0}$ is the angle between the chain tangential vectors at the intersection $a$.

form (cf. [16] and Eqs. $(2.2,2.6))$ :

$$
\mathcal{F}_{\text {int }}=\mathcal{F}-\mathcal{F}_{\text {Ideal }} \simeq \frac{1}{V}\left\{-\ln \int \mathrm{e}^{-E} \mathrm{~d} X_{f(\mathbf{n})}+\ln \int \mathrm{d} X_{f(\mathbf{n})}\right\}
$$

where $V$ is the total volume of the system, $E=\sum_{a} U_{a}$ is the total interaction energy of the system, $U_{a}$ is the interaction energy of a pair of fragments of length $\lambda$ (defined in Sect. 3), and the index $a$ runs over all possible pairs. The integration in the conformational space (over $\mathrm{d} X_{f(\mathbf{n})}$ ) is restricted to a given orientational order described by the probability distribution $f(\mathbf{n})$.

Using the notation

$$
\beta_{a} \equiv 1-\mathrm{e}^{-U_{a}}
$$

(cf. Eq. (2.6)) we can write the interaction energy per unit volume as:

$$
\begin{aligned}
\mathcal{F}_{\text {int }} & \simeq \mathcal{F}_{\beta}+\mathcal{F}_{\beta^{2}}+. . \\
\mathcal{F}_{\beta} & =\frac{1}{V} \sum_{a}\left\langle\beta_{a}\right\rangle_{f(\mathbf{n})} ; \\
\mathcal{F}_{\beta^{2}} & =-\frac{1}{V} \sum_{a, b ; a \neq b}\left(\left\langle\beta_{a} \beta_{b}\right\rangle_{f(\mathbf{n})}-\left\langle\beta_{a}\right\rangle_{f(\mathbf{n})}\left\langle\beta_{b}\right\rangle_{f(\mathbf{n})}\right)
\end{aligned}
$$

where $a, b, .$. are the indices of the different pairs of fragments, and the average $\langle\ldots\rangle$ corresponds to an integration over all possible positions of the segments of all the chains for a given distribution $f(\mathbf{n})$. The first term in the expansion (4.3) gives the mean field result, i.e. the local second virial interaction term: $\mathcal{F}_{\beta} \simeq \nu^{2} B_{\lambda} / 2$ (cf. with Eq. (3.4)).

The next term in equation (4.3), $\mathcal{F}_{\beta^{2}}$, which is quadratic in $\beta_{a}$, gives the correlation corrections to the mean field second virial coefficient. In principle one can also include higher order virial terms, however the main contribution to the interaction free energy comes from the correlation correction to the second virial coefficient. Below we estimate the variation of this correlation term $\mathcal{F}_{\beta^{2}}$ with the orientational order and concentration.

The segments of the chains interact via a steric potential: the energy $E$ vanishes provided that no segments intersect in space, it is infinite if one pair of segments (or more) does intersect. Hence

$$
\beta_{a}=\left\{\begin{array}{lc}
1, & \text { if this pair of segments intersects; } \\
0, & \text { otherwise. }
\end{array}\right.
$$

If the pairs " $a$ " and " $b$ " belong to different chains, the intersections are nearly independent and thus do not contribute to $\mathcal{F}_{\beta^{2}}$. However, if the two pairs belong to the same pair of chains (see Fig. 3), their contribution to $\mathcal{F}_{\beta^{2}}$ may be important. Indeed, if the pairs " $a$ " and " $b$ " belong to the same chains and are very close to each other, the contact " $a$ " implies that the contact " $b$ " takes place with probability of order unity, and so $\left\langle\beta_{a} \beta_{b}\right\rangle \simeq\left\langle\beta_{a}^{2}\right\rangle=\left\langle\beta_{a}\right\rangle \sim \nu B_{\lambda}$, whereas 
$\left\langle\beta_{a}\right\rangle\left\langle\beta_{b}\right\rangle=\left\langle\beta_{a}\right\rangle^{2} \sim \nu^{2} B_{\lambda}^{2}$, which is much lower than $\nu B_{\lambda}$ provided that the concentration $\nu$ is low enough.

Clearly it is the situation shown in Figure 3 that gives the main contribution to the correlation correction term, $\mathcal{F}_{\beta^{2}}$, given by equation (4.4). It is also clear that the correction depends on the internal chain statistics only and is not essentially influenced by the global concentration $\nu$ (the orientational distributions of the chains are fixed by the distribution function $f(\mathbf{n})$ ). Below we estimate the ratio $\mathcal{F}_{\beta^{2}} / \mathcal{F}_{\beta}$ at low concentrations $(\nu \rightarrow 0)$. If the two segments of a pair " $a$ " are not in contact, $\beta_{a}=0$ and this pair does not contribute neither to $\mathcal{F}_{\beta}$, nor to $\mathcal{F}_{\beta^{2}}$. Only the pairs of segments in contact $\left(\beta_{a}=1\right)$ contribute to the ratio $\frac{-\mathcal{F}_{\beta^{2}}}{\mathcal{F}_{\beta}}$ As explained above, we also must sum the correlation contributions over the pairs " $b$ " that belong to the same chains as the pair " $a$ " so that

$$
\left.\frac{-\mathcal{F}_{\beta^{2}}}{\mathcal{F}_{\beta}} \simeq \frac{\sum_{b}\left\langle\beta_{a} \beta_{b}\right\rangle}{\left\langle\beta_{a}\right\rangle}\right|_{\beta_{a}=1}=\left.\sum_{b}\left\langle\beta_{b}\right\rangle\right|_{\beta_{a}=1} \equiv \mathcal{N} .
$$

Thus, the ratio of the correlation free energy $\mathcal{F}_{\beta^{2}}$ to the standard mean field contribution $\mathcal{F}_{\beta}$ is just the number $\mathcal{N}$ of (secondary) contacts between the crossing chains, i.e. the number of contacts " $b$ " which "coat" a given contact " $a$ ". Note the negative sign of the ratio $\mathcal{F}_{\beta^{2}} / \mathcal{F}_{\beta}$ (Eq. (4.6)), its origin can be understood as follows. The mean field result $\mathcal{F}_{\beta}$ is positive and it comes from excluded volume interactions between all pairs of segments taken independently. In the mean-field picture the situations with two contacts between the chains are excluded twice from the partition function, thus $\mathcal{F}_{\beta}$ over-estimates the real losses in configurational space due to interactions, i.e. over-estimates the free energy. Hence the correction $\left(\mathcal{F}_{\beta^{2}}\right)$ to the mean field result should be negative (for more details see the last section, Eqs. $(5.7,5.8)$ ).

\subsection{Correlation Corrections for the Case of Ordinary Persistent Chains. -}

In order to illustrate the method we start with the case of ordinary semiflexible chains $\left(l_{2}=0\right)$. We consider the nematic $N$-phase with a characteristic orientation angle $\gamma$. Suppose that a pair " $a$ " (of segments which belong to chains " 1 " and " 2 ") is in contact and that the two segments cross at an angle $\gamma_{0} \leq \gamma$. We want to estimate the probability that a segment " $\lambda$ " at a distance $x$ from the pair " $a$ " along the chain " 1 " has a contact with the other chain "2" (see Fig. 3), and the typical number of such contacts between the chains " 1 " and " 2 ". If $x \lesssim \gamma_{0}^{2} l$, there is no contact because the chains are relatively stiff at these scales and so they can be considered as rigid rods which do not meet twice. If $\gamma_{0}^{2} l \lesssim x \lesssim \gamma^{2} l$ the chains are already flexible, and the segment " $\lambda$ " occupies a typical volume $V_{x} \sim \lambda\left(x \gamma_{\text {ch }}\right)^{2}$, where $\gamma_{\mathrm{ch}} \sim(x / l)^{05} \ll 1$, which includes the trajectory of the chain "2"; the probability $p_{x}$ of their intersection is of order $B_{x} / V_{x}$, where the typical excluded volume is $B_{x} \sim \lambda^{2} d \gamma_{c h}$ (note that the length of the segments is $\lambda)$. Hence $p_{x} \sim \lambda d /\left(x^{2} \gamma_{\mathrm{ch}}\right) \sim \lambda d l^{1 / 2} x^{-5 / 2}$, and the total number of secondary intersections between the chains is $\mathcal{N} \simeq \int p_{x}(\mathrm{~d} x / \lambda) \sim d l^{1 / 2} / x_{\min }^{3 / 2}$. The lower cutoff in the integral for $\mathcal{N}$, which diverges at the lower limit, is $x_{\min } \simeq \gamma_{0}^{2} l$. To describe the typical situation in the nematic phase we should put $\gamma_{0} \simeq \gamma$. The typical number of secondary contacts in the nematic phase formed by semiflexible chains is therefore:

$$
\mathcal{N} \simeq \frac{d}{l \gamma^{3}}
$$

The interaction free energy including the correlation corrections can be thus approximately written as $\mathcal{F}_{\text {int }} \simeq \mathcal{F}_{\beta}(1-\mathcal{N})$, where $\mathcal{F}_{\beta}$ is the ordinary interaction energy in the mean field approximation (see Eqs. $(4.3,4.6)$ ). 
The total free energy (per chain) for solution of semiflexible chains in the mean field approximation can be written in analogy with equation (3.5):

$$
\mathcal{F} \sim \ln \Phi+\ln \left(\frac{1}{\Gamma}\right)+\frac{L}{l} \Gamma^{-2}+\Phi \frac{L}{d} \Gamma
$$

where $\Gamma=\sin \gamma$ and numerical coefficients are omitted. In accordance with (4.7), with correlation corrections the free energy becomes

$$
\mathcal{F} \sim \ln \Phi+\ln \left(\frac{1}{\Gamma}\right)+\frac{L}{l} \Gamma^{-2}+\Phi \frac{L}{d} \Gamma\left(1-\frac{d}{l \Gamma^{3}}\right) .
$$

The estimations of the correlation corrections are made above for long enough chains: $L \gtrsim x_{\min } \gtrsim l \gamma^{2}$, hence the second term in the sum (4.9) is negligible in comparison with the third one.

The correlation corrections could become important in the nematic phase, if the orientation angle is small enough:

$$
\gamma \lesssim(d / l)^{1 / 3}
$$

The typical orientation angle is determined by the balance of the entropic third term and the mean field virial term:

$$
\gamma \sim\left(\frac{d}{l \Phi}\right)^{1 / 3}
$$

Hence, the condition (4.10) is impossible to fulfill, as it corresponds to solutions with a volume fraction $\Phi \gtrsim 1$, and so the correlation corrections are always small for solutions of ordinary semiflexible chains.

It should be mentioned that in the calculation of the number of secondary contacts $\mathcal{N}$, we have omitted an upper limit divergence in the integral. Let us consider an isotropic solution of semiflexible chains $(\gamma=\pi / 2)$. Very distant segments with $x \gtrsim l$ are effectively confined in a sphere with radius of order $R_{x} \sim(x l)^{1 / 2}$ so that their typical concentration is $\nu_{x} \sim(x / \lambda) R_{x}^{-3}$ Therefore the intersection probability for a segment is $p_{x} \sim v_{x} \lambda^{2} d \sim \lambda d\left(x l^{3}\right)^{-1 / 2}$, which leads to the upper limit divergence of the integral for

$$
\mathcal{N} \simeq \int\left(p_{x} / \lambda\right) \mathrm{d} x \sim\left(\mathrm{d} x_{\max }^{1 / 2} l^{-3 / 2}\right)
$$

However, this divergence is the standard consequence of the excluded volume interactions which lead to the chain swelling. In a concentrated or semidilute system all large scale interactions (in particular, large scale contributions to $\mathcal{N}$ ) are screened on scales larger than effective correlation length. In turn, this effective correlation length is the size of interaction blob (the chain fragment of length $\Lambda$ with typical interaction energy of order 1 ) or the contour length of the chain $L$, whichever is smaller:

$$
\tilde{\Lambda} \equiv \min \{\Lambda, L\} .
$$

Thus the upper limit in the integral in equation (4.12) should be set to $x_{\max } \sim \tilde{\Lambda}$. In the mean-field regime when the correlation corrections are small we get using equation (4.8) $\Lambda \sim d /(\Phi \Gamma)$. Using equation (4.12) with $x_{\max } \sim \tilde{\Lambda}$ it is straightforward to check that the "upper limit" correlation correction is indeed small if $\Phi \gtrsim(d / l)^{3}[17,18]$ Thus for the nematic regime $(\Phi \gtrsim d / l)$ the correlation corrections are always negligible. 
4.3. Correlation Corrections for the Polymers with Planar Rigidity. - Let us now proceed to study solutions of chains with anisotropic rigidity and calculate the "lower limit" correlation corrections to the last term of the free energy (Eq. (3.5)). As we show in paper I, the statistics of the chains with two rigidities depends on the value of the parameter $\Omega$ (Eq. (1.2)). Let us start with the regime $(d / l)^{2} \lesssim \Omega \lesssim 1$ (i.e. $l<l_{2}<L_{4}$ ) and consider a $\mathrm{P}$-phase with the characteristic orientation angle $\psi$.

We consider two chains with their PMFs crossing at an angle $\psi_{0} \leq \psi$, and with a contact at point " $a$ ". Using the same procedure as above for semiflexible chains, we can calculate the typical probability $p_{x}$ of a contact between the segment " $\lambda$ " of one chain with the other chain (the segment " $\lambda$ " lays at a contour length $x$ from the point " $a$ "). The result is:

$$
p_{x} \sim\left\{\begin{array}{ccc}
0, & \text { if } & x \lesssim l ; \\
d \lambda /\left(x^{1 / 2} l^{3 / 2} \psi_{0}\right), & \text { if } & l \lesssim x \lesssim \psi_{0}^{2} l_{2} \\
\Omega^{1 / 2} \lambda / x, & \text { if } & \psi_{0}^{2} l_{2} \lesssim x \lesssim l_{2} \\
d \lambda /\left(x^{1 / 2} l^{3 / 2}\right), & \text { if } & l_{2} \lesssim x \lesssim L_{4}
\end{array}\right.
$$

For $x \lesssim l$ the chain fragments of length $x$ are rod-like (note that the typical angle of their intersection is $\gamma \sim 1$ ), hence the secondary contacts are absent. For $l \lesssim x \lesssim \psi_{0}^{2} l_{2}$ the dimensions of the fragments are $R_{\|} \sim(l x)^{1 / 2}$ and $R_{\perp} \sim x\left(l / l_{2}\right)^{1 / 2}$ (cf. Eq. (I.3.12), Fig. I.3b from paper I), their typical volume is $V_{x}^{\prime} \sim R_{\|}^{2} R_{\perp}$, and the typical segment concentration inside the fragments is $\nu_{x}=(x / \lambda) / V_{x}$. The corresponding volume of the intersection between fragments is $V_{\cap}=R_{\|} R_{\perp}^{2}\left(\sin \psi_{0}\right)^{-1}$ The probability of a secondary contact is thus $p_{x}=\nu_{x} \lambda^{2} d\left(V_{\cap} / V_{x}\right) \sim$ $d \lambda /\left(x^{1 / 2} l^{3 / 2} \psi_{0}\right)$.

For $\psi_{0}^{2} l_{2} \lesssim x \lesssim l_{2}$ (or, if $\psi_{0}<\left(l / l_{2}\right)^{1 / 2}$, for $l \lesssim x \lesssim l_{2}$ ) the initial angle between the PMFs of the chains is small in comparison with the typical change of the PMF's orientation on the scale $x: \psi_{0} \ll \Delta \psi(x) \sim\left(x / l_{2}\right)^{1 / 2}$ (see Eq. (I.2.5)). Similar arguments give $V_{\cap}=R_{\|}^{2} R_{\perp} \sim V_{x}$, and $p_{x}=\nu_{x} \lambda^{2} d \sim\left(l_{2}^{1 / 2} d \lambda\right) /\left(x l^{3 / 2}\right)$.

At larger scales $\left(x \gtrsim l_{2}\right)$ the fragments $x$ are isotropic $\left(R_{\|} \sim R_{\perp} \sim(l x)^{1 / 2}\right)$, and $p_{x}=d \lambda /\left(x^{1 / 2} l^{3 / 2}\right.$ ), hence $\mathcal{N} \sim x^{1 / 2} d / l^{3 / 2} \propto x^{1 / 2}$ ( quite similarly to what was explained above for semiflexible chains with $x \gtrsim l$ ). However this "upper limit" divergence is related to global chain swelling effect and is not important here.

From the intersection probability $p_{x}$ (Eq. (4.14)), we can get the typical number of secondary contacts by integration: $\mathcal{N} \simeq \int_{0}^{\tilde{\Lambda}}\left(p_{x} / \lambda\right) \mathrm{d} x, \tilde{\Lambda}=\min \{\Lambda, L\}$ where $\Lambda$ is the contour length of the effective interaction blob (see the end of subSect. 4.2). From equation (3.5) one can easily find that $\Lambda \simeq d / \Phi$ and that $\Lambda \lesssim \psi^{2} l_{2}$. On the other hand, for the P-phase symmetry we should have $\Phi \lesssim d / l$ (otherwise a B-phase will be formed, see discussion in paper I, Sect. I.4.1), hence $\Lambda \gtrsim l$. Thus the final result for the case $(d / l)^{2} \lesssim \Omega \lesssim 1$ is

$$
\mathcal{N} \simeq \frac{\tilde{\Lambda}^{1 / 2} d}{l^{3 / 2} \psi}, \text { with } \tilde{\Lambda} \equiv \min \{\Lambda, L\}
$$

where we take into account that typically $\psi_{0} \sim \psi$ in the P-phase. 
In the case of large second rigidity $l_{2}>L_{4}(\Omega \gtrsim 1)$ similar arguments show that

$$
p_{x} \sim\left\{\begin{array}{ccc}
0, & \text { if } & x \lesssim l ; \\
d \lambda /\left(x^{5 / 4} l^{3 / 4} \psi_{0}\right), & \text { if } & l \lesssim x \lesssim L_{5} \\
d \lambda \Omega^{-3 / 10} x^{-1 / 2} l^{-3 / 2} \psi_{0}^{-1}, & \text { if } & L_{5} \lesssim x \lesssim \psi_{0}^{2} l_{2} \\
\Omega^{1 / 5} \lambda / x, & \text { if } & \psi_{0}^{2} l_{2} \lesssim x \lesssim l_{2} \\
\lambda\left(d^{2} l^{-3} x^{-4}\right)^{1 / 5}, & \text { if } & x \gtrsim l_{2}
\end{array}\right.
$$

if $\Omega \gtrsim 1$ and $\psi_{0} \gtrsim d / l$.

Again we assume that $\psi_{0} \sim \psi$, take into account that the unequalities $l \lesssim \Lambda \lesssim l_{2} \psi_{0}^{2}$ are still valid (see Eq. (3.5)), hence only the second and the third lines of equation (4.16) are important, and thus the number of contacts is:

$$
\mathcal{N} \simeq \frac{1}{\psi}\left[\frac{d}{l}+\left(\frac{\tilde{\Lambda}}{L_{6}}\right)^{1 / 2}\right], \quad \text { if } \quad \Omega \gtrsim 1 \quad \text { and } \quad \psi \gtrsim d / l .
$$

For smaller angles between the disks, $\psi<d / l$, the scaling approach outlined in Appendix A should be used. The general result valid in both regimes $(\psi>d / l$ and $\psi<d / l)$ for $\Omega \gtrsim 1$ is

$$
\mathcal{N} \simeq \frac{1}{\psi}\left(\frac{\tilde{\Lambda}}{L_{6}}\right)^{1 / 2}+\varphi\left(\frac{d}{l \psi}\right)
$$

where $\varphi(x) \sim\left\{\begin{array}{cl}x & , \quad x \ll 1 \\ x^{2 / 3} & , \quad x \gg 1\end{array}\right.$.

Taking into account the correlation corrections, we rewrite the interaction contribution (per chain) to the free energy (3.5) in the P-phase $(\gamma=\pi / 2)$ :

$$
\mathcal{F}_{\text {int }} \simeq \Phi \frac{L}{d} b(\Psi)(1-\mathcal{N}) \quad \text { for P-phase }
$$

where $\mathcal{N}$ is determined by equations $(4.15,4.17)$. It should be mentioned that the correlation corrections bring an additional angular dependence into the free energy functional $(3.5,4.19)$. These $\psi^{-1}$-like dependencies can compete with other angular dependent terms in the free energy (3.5). Note, that their sign is negative, so that the correlation corrections lead to an additional ordering of the polymers in the P-phase. The origin of this effect is rather clear: for very rigid disc-like chains (if $l_{2}$ is large) the higher the order parameter in the P-phase, the higher the probability of secondary contacts (this means that the apparent excluded volume is smaller that the one calculated from the pure mean field approximation).

We have calculated here the correlation corrections when they are small i.e. when the average number of secondary contacts is smaller than one. A more general discussion of the secondary contacts is given in the discussion section.

\section{Discussion and Conclusions}

In this paper we have applied the Onsager theory based on the second virial approximation to investigate nematic ordering in solutions of chains with anisotropic rigidity. Various generalizations of this method have also been proposed. 
In Section 2 a classical expansion over the coil concentration (the "global" approach) was introduced in the case where the shape of the coils is anisotropic. The result can be applied to study the pancake nematic ( $P$-phase). We have explicitly calculated the pair interaction energy and the virial coefficient between anisotropic ribbon polymer chains. Both a mean field approximation (Appendix B) and a scaling appraach (Appendix A) were introduced. The higher order terms in the virial expansion are estimated in Appendix $\mathrm{C}$ where we show that the second virial approximation is almost always sufficient.

For higher concentrations or when the polymer chains are rather long ( $L \gtrsim l_{2}$, so that the coils are globally isotropic) another extension of the second virial approximation based on the mean field approach is proposed (see Sect. 3). The polymer solution is considered as a solution of short fragments connected into chains with particular statistics reflecting the polymer structure with two rigidities. The free energy of nematic phases with various symmetries ( $\mathrm{P}$ - or $\mathrm{N}$-type) has been calculated using this "local" approach.

The internal two-dimensional-like statistics of the coils induces strong correlations which lead to corrections to the "bare" virial coefficients calculated in Section 3. These correlation corrections are discussed in Section 4. Unlike the case of ordinary semiflexible chains (discussed in Sect. 4.2), in the case of polymers with two rigidities the correlation corrections can contribute significantly to the free energy.

In order to clarify the relationship between the local and the global approaches, let us consider the case of a dilute solution when the second virial approximation is definitely valid. The second virial coefficient between two chains can be exactly defined as

$$
B=\int\left(1-e^{-E}\right) \mathrm{d}^{3} R
$$

where $\mathbf{R}$ is the vector connecting centres of mass of two coils, and $E(\mathbf{R})=\sum_{a} U_{a}$ is the total interaction energy with $a$ running over all possible pairs of segments belonging to different chains (in addition the r.h.s. of Eq. (5.1) should be averaged over all possible conformations of the two chains; this averaging is assumed but not shown explicitly in order to simplify the notations). Using segmental Mayer functions defined in equation (4.2) we get,

$$
B=\int\left[1-\prod_{a}\left(1-\beta_{a}\right)\right] \mathrm{d}^{3} R=\int \sum_{a} \beta_{a} \mathrm{~d}^{3} R-\int \sum_{a>b} \beta_{a} \beta_{b} \mathrm{~d}^{3} R+. .
$$

Obviously the 1st term in the r.h.s. of equation (5.2) is the sum of local contributions of all pairs of segments:

$$
B_{\text {loc }}=\int \sum_{a} \beta_{a} \mathrm{~d}^{3} R
$$

Omitting all higher order terms but this first term we get the free energy of interaction which is exactly equal to the outcome of the local approach (Eq. (3.4)):

$$
\mathcal{F} \simeq \frac{c}{2} B_{\text {loc }}
$$

(per chain). The second and higher order terms in the r.h.s. of equation (5.2) represent the corrections to $B_{\text {loc }}$ due to the existence of 2 or more contacts between the segments of the two chains. In other words the higher order terms account for additional contacts implied by primary contacts. Therefore these terms corresponds exactly to the correlation corrections to the local approximation calculated in Section 4.

The correlations corrections are small if the situations with 2 and more contacts are rare, i.e. if the averaged number of contacts is small. This condition means that the typical effective 
free energy $U$ of interaction between the chains is small: $U \ll 1$. In a more concentrated (semidilute) system a similar condition can be formulated with $U$ being a the free energy of interaction between two interaction blobs. Since by definition the total interaction energy per blob is $\sim 1$, the condition $U \ll 1$ means that the number of interaction blobs overlapping a given blob is large. Therefore the local approximation is essentially a mean-field approximation.

In Section 4 we showed that with correlation correction the interaction free energy is (see Eq. (4.19))

$$
B \simeq B_{\text {loc }}(1-\mathcal{N})
$$

where $\mathcal{N}$ is the number of secondary contacts which is assumed to be small, $\mathcal{N} \ll 1$. With the global approach the second virial coefficient is defined by equation (2.6):

$$
B \simeq \int\left(U-U^{2} / 2\right) \mathrm{d}^{3} R
$$

Physically the second term in brackets in equation (5.6) corresponds to the second correction term in equation (5.5). One can check e.g. for $\Omega \lesssim 1$ (when the mean field approximation is appropriate) that with equation (B.5) for $U$ and equation (4.15) for $\mathcal{N}(\tilde{\Lambda}=L)$ these terms are identical.

For a system with steric interactions only (athermal system) it is possible to find a general relation between $B$ and $B_{\text {loc }}$. Note that integrands in equations (5.1) and (5.3), $\beta=1-\mathrm{e}^{-E}$ and $\beta_{\mathrm{loc}}=\sum_{a} \beta_{\mathrm{a}}$ are both equal to 0 if there are no contacts between the two chains. On the other hand if the chains intersect, then $\beta=1$ and $\beta_{\text {loc }}$ is equal to the total number of contacts between the chains: $\beta_{\text {loc }}=1+\mathcal{N}$, counting one primary contact and $\mathcal{N}$ secondary contacts. Therefore

$$
B=B_{\text {loc }} /(1+\mathcal{N}) \text {. }
$$

This equation is generally valid: it does not require $\mathcal{N}$ to be small. For $\mathcal{N} \ll 1$ equation (5.7) agrees with equation (5.5). Thus we get the following general equation for the free energy of interaction in the second virial approximation which is always valid (within the scaling accuracy):

$$
\mathcal{F}_{\text {int }}=\mathcal{F}_{\text {int }}^{\text {loc }} /(1+\mathcal{N})
$$

where $\mathcal{F}_{\text {int }}^{\text {loc }}$ is the interaction free energy within the local second virial approximation defined by equation (3.4), and $\mathcal{N}$ is the average number of secondary contacts between two interacting chains of contour length $L$ (in the dilute regime) or between two overlapping interaction blobs $\Lambda$ (in the semidilute regime). It is important to note that the number of secondary contacts, $\mathcal{N}$, should be calculated with primary conformations of two chains which are unperturbed by the interaction between the chains.

Equation (5.8) is valid in all the dilute and semidilute regimes including the situations where the global approach is also applicable. This statement is further illustrated in Appendix A.

In conclusion, the local approach developed in Section 3 (together with correlation corrections found in Sect. 4) is the most general and powerful version of Onsager-like theories discussed in the present paper. In particular, to describe P-nematic type ordering in a ribbon polymer solution one can use the following scaling equation for the free energy (per chain) (see Eqs. $(2.8,3.5,5.8)$ )

$$
\mathcal{F}=\ln \Phi+\ln \left(\frac{1}{\Psi}\right)+\frac{L}{l_{2}} \Psi^{-2}+\Phi \frac{L}{d} \frac{\left[1+\Psi^{2}\right]}{[1+\mathcal{N}]}
$$

where the number of secondary contacts $\mathcal{N}$ can be estimated with the help of equation (4.15) (if $(d / l)^{2} \lesssim \Omega \lesssim 1$ ) or equation (4.18) (if $\Omega \gtrsim 1$ ).

The methods developed in this paper will be used in the next (third) paper of this series to study liquid crystalline ordering in solutions of ribbon-like chains [20]. 

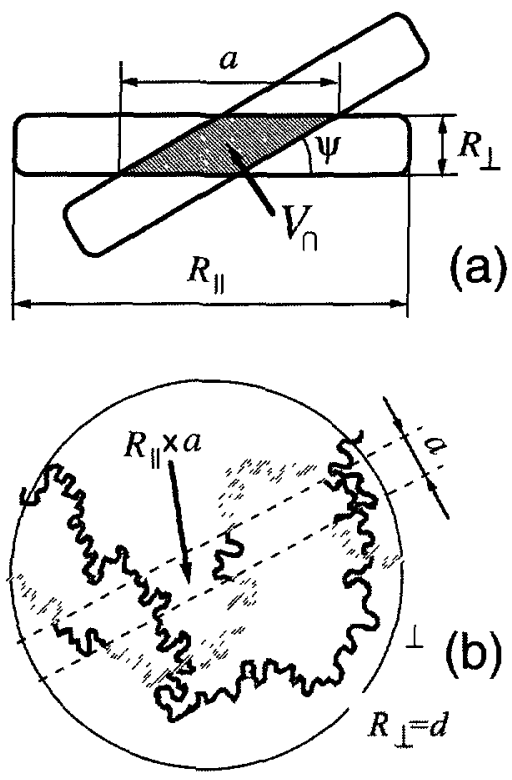

Fig. 4. - (a) Two overlapping discs; $V_{\cap}$ is the volume of intersection, $a=R_{\perp} / \sin \psi$. (b) Another projection for the case $R_{\perp}=d$ (flat coil regime): the dark grey chain interacts with the light grey chain; the fragments of the second chain which participate in the interaction are shown in black.

\section{Acknowledgments}

This work was supported by CNRS (France), by NATO's Scientific Affairs Division in the framework of the Science for Stability Programme and by EPSRC grant GR/L37694 (U.K.).

\section{Appendix A}

\section{Interaction Energy of Crumbly (Loose) Discs in the Scaling Regime}

In this appendix we calculate the second virial coefficient of two swollen disk-like coils (the regime $\Omega>1$ described in Sect. 3.3 of Ref. [4]) using both the global and the local approaches. For simplicity we adopt the scaling accuracy here and neglect all corrections to $B$ if these are formally small, no matter whether these corrections are important for liquid crystalline ordering or not.

We start with the global approach. According to the results of paper I (Sect. I.3.2), an isolated chain with two rigidities $l$ and $l_{2}$ has a disc-like shape $\left(R_{\|} \gg R_{\perp}\right)$, if its contour length $L$ is not too long: $l \lesssim L \lesssim l_{2}$. When two such discs intersect, they repel each other because of the excluded volume interactions. For a given angle between the orientations of the chains, $\psi$, the typical volume of the discs intersection is

$$
V_{\cap} \sim \frac{R_{\|} R_{\perp}^{2}}{\sin \psi+R_{\perp} / R_{\|}}
$$

(see Fig. 4a). This is the volume where the links of the two chains interact. 
We first estimate the interaction energy $U$ in the case where the scaling "blob" picture described in Section I.3.3 is valid, i.e. for $\Omega \gtrsim 1$. We start from the case of "flat swollen coils" ( $l \lesssim L \lesssim L_{5}$ ), when $R_{\perp} \simeq d$ and $R_{\|} \sim\left(l L^{3}\right)^{1 / 4} \quad$ In this case if $\psi \gtrsim d / R_{\|}$then the intersection region $V_{n}$ is a stripe of width $a=d / \sin \psi$ (cf. Figs. 4a, b) and of area $a R_{\|}$(the total area of the discs being of order $\left.R_{\|}^{2} \sim\left(L^{3} l\right)^{1 / 2}\right)$. The links belonging to this stripe directly contribute to the interactions between the chains (see Fig. 4b). Hence, the interaction energy between the two chains is the interaction energy between the fragments of the chains inside the stripe. The size of these fragments in the disc plane is $a$. If the angle $\psi$ between the PMFs of the chains is small enough $(\psi \lesssim d / l$, and hence $a \gtrsim l)$, the fragments inside the stripe are "flat swollen blobs" with contour length $\lambda \sim l(a / l)^{4 / 3} \gtrsim l$; for larger angles $\psi$, they are the rod-like fragments of typical length $\lambda \sim a \lesssim l$. In both cases the total contour length of the fragments inside the stripe is $\sim L\left(a / R_{\|}\right)$, hence the number of $\lambda$-fragments in the stripe is $m_{a} \sim\left(L a / R_{\|}\right) / \lambda$, their characteristic two-dimensional concentration is $\sim m_{a} /\left(a R_{\|}\right)$, and their interaction energy is $U \sim m_{a}^{2} a^{2} /\left(a R_{\|}\right)$, where we used the fact that the typical "excluded area" of the $\lambda$-fragments is of order $a^{2}$ (which is true both for the flat swollen blobs and for the rod-like fragments). Thus

$$
U \sim \frac{L^{2} a^{3}}{\lambda^{2} R_{\|}^{3}} \sim\left(\frac{l}{L}\right)^{1 / 4} \times\left\{\begin{array}{cc}
\left(\frac{d}{l \sin \psi}\right)^{1 / 3}, & \text { if } \frac{d}{\left(L^{3} l\right)^{1 / 4}} \lesssim \sin \psi \lesssim \frac{d}{l} \\
\frac{d}{l \sin \psi}, & \text { if } \sin \psi \gtrsim \frac{d}{l}
\end{array}\right.
$$

$$
\left(\text { for } \Omega \gtrsim 1 ; \quad l \lesssim L \lesssim L_{5}\right) \text {. }
$$

Note, that in the case of larger angles $\psi(\psi \gtrsim d / l)$, when the $\lambda$-fragments are rod-like, equation (A.2) coincides with the corresponding result obtained in Appendix B using the local approach (the mean field approximation) (Eq. (B.5)).

Using equations (2.6) and (A.2) we get the second virial coefficient

$$
B(\psi) \sim\left\{\begin{array}{cc}
d R_{\|}^{2}, & \psi<d / R_{\|} \\
R_{\|}^{3} \psi\left(\frac{l}{L}\right)^{1 / 4}\left(\frac{d}{l \psi}\right)^{1 / 3}, & d / R_{\|}<\psi<d / l . \\
L^{2} d, & \psi>d / l
\end{array}\right.
$$

Let us now consider the case of longer chains ( $L_{5} \lesssim L \lesssim l_{2}, \Omega \gtrsim 1$ ), when the "body" of the chain (of the corresponding disc-like coil) is thicker than $d$, but is still anisotropic: $d<R_{\perp}<R_{\|}$, where $R_{\perp} \sim(L d / l) \Omega^{-2 / 5}, R_{\|} \sim(L l)^{1 / 2} \Omega^{1 / 10}$ In the framework of the scaling picture described in Section I.3.3, for $L_{5} \lesssim L \lesssim l_{2}$ each chain can be represented as a "monisto" of coins of width $d$ and diameter $R_{5}$. The sequence of coins is fully stretched in the direction perpendicular to the PMF of the chain, so that the coins interact nearly independently. The interaction energy for each pair of coins can be estimated as described above for the case of "flat" chains. Each chain includes $R_{\perp} / d \simeq L / L_{5}$ coins. When two chains intersect with the intersection angle $\psi \gtrsim R_{\perp} / R_{\|} \sim\left(L / l_{2}\right)^{1 / 2}$, the intersection volume is $V_{\cap} \sim R_{\|} a^{2} \sin \psi \sim R_{\|} R_{\perp}^{2} / \sin \psi$. The probability $p$ that two coins (belonging to these chains) intersect is $p=p_{1}^{2} p_{2}$, where $p_{1} \sim V_{\cap} /\left(R_{\perp} R_{\|}^{2}\right)$ is the probability to find a coin inside $V_{\cap}$, and $p_{2} \sim R_{5}^{3} \sin \psi / V_{\cap}$ is the probability that the coins intersect once they are inside $V_{\cap}$. Thus $p \sim R_{5}^{3} / R_{\|}^{3}$. The interaction energy of two intersecting coins, $U_{L_{5}}$, is determined by 
equation (A.2) with $L \simeq L_{5}$. So the total interaction energy between the two chains is

$$
U \sim\left(\frac{L}{L_{5}}\right)^{2}\left(\frac{R_{5}}{R_{\|}}\right)^{3} U_{L_{5}} \sim\left(\frac{L}{l}\right)^{1 / 2} \Omega^{-3 / 10} \times\left\{\begin{array}{ccc}
\left(\frac{d}{l \sin \psi}\right)^{1 / 3}, & \text { if } & \left(\frac{L}{l_{2}}\right)^{1 / 2} \lesssim \sin \psi \lesssim \frac{d}{l} \\
\frac{d}{l \sin \psi}, & \text { if } & \sin \psi \gtrsim \frac{d}{l}
\end{array}\right.
$$

$$
\text { (for } \left.\Omega \gtrsim 1 ; \quad L_{5} \lesssim L \lesssim l_{2}\right)
$$

Note again that in the case of large enough angles,

$$
\psi \gtrsim \psi_{\mathrm{mf}} \simeq d / l
$$

(i.e. when the second lines of Eqs. (A.2, A.4) are to be used), the $\lambda$-blobs are not swollen, so that the mean-field approximation is valid: the above results coincide with equation (B.5) derived below in Appendix B. However, in equations (A.2, A.4) the numerical coefficient $b(\psi)$ (of order unity), which is considered in more details in Appendix B, is omitted.

With equations (2.6, A.4) we get finally:

$$
B(\psi) \simeq R_{\|}^{2} R_{\perp}+\min \left[R_{\|}^{3} \psi, L^{2} d^{1 / 3}(\psi l)^{2 / 3}, L^{2} d\right]
$$

Note that equation (A.6) is general: it is also valid for shorter chains, $L<L_{5}$, when $R_{\perp}=d$.

So far we have considered the second virial coefficient $B(\psi)$ within the global approach which is needed for the calculations in Section 2. Let us now implement briefly the local approach discussed in Section 5 (Eq. (5.7)) and demonstrate that it gives the same result (A.6) as the global approach. As shown in Appendix B

$$
B_{\mathrm{loc}} \sim L^{2} d
$$

In accordance with equation (5.7) the local virial coefficient should be renormalized by the factor $1 /(1+\mathcal{N})$. We consider here only the cases where $\mathcal{N} \gg 1$, i.e. when such a renormalization is essential. Next we note two contributions to the number of secondary contacts, $\mathcal{N}$, coming from the scales $\sim \lambda$ and $\sim L: \mathcal{N}=\mathcal{N}_{\lambda}+\mathcal{N}_{L}$, where $\mathcal{N}_{L} \simeq \frac{1}{\psi}\left(\frac{L}{L_{6}}\right)^{1 / 2}$ for $L \gtrsim L_{5}$ (it can be calculated using the 3rd line of equation (4.16) (cf. Eq. (4.17)). For $\psi>d / l$ the local contribution $\mathcal{N}_{\lambda}$ can be calculated using the second line of equation (4.16): $\mathcal{N}_{\lambda} \simeq \frac{d}{l \psi}$, and thus it is small: $\mathcal{N}_{\lambda}<1$. For smaller angles, $\psi<d / l$, the local contribution to $\mathcal{N}$ is equal to the number of contacts between two overlapping flat (and 2d swollen) $\lambda$-blobs: $\mathcal{N}_{\lambda} \simeq \lambda^{2} / a^{2}$, where $a=l(\lambda / l)^{3 / 4}=d / \psi$ is the spatial size of a $\lambda$-blob; thus we get $\mathcal{N}_{\lambda}=(d / \psi l)^{2 / 3}$ in agreement with equation (4.18). So we get

$$
\mathcal{N}=\mathcal{N}_{\lambda}+\mathcal{N}_{L} \simeq \min \left\{\left(\frac{d}{l \psi}\right)^{2 / 3}, \frac{d}{l \psi}\right\}+\frac{1}{\psi}\left(\frac{L}{L_{6}}\right)^{1 / 2}
$$

Now using equations (5.7, A.7, A.8) we recover the "global" result (Eq. (A.6)) for the second virial coefficient in the regime $\psi>R_{\perp} / R_{\|}$. 


\section{Appendix B}

\section{The Second Virial Coefficient between Crumbly Discs in the Local (Mean Field) Approximation}

We now calculate in more detail the interaction energy, $U$, and the second virial coefficient of two crumbly (loose) dises (made of chains with two rigidities $l$ and $l_{2}$ ) in the mean field approximation, i.e. neglecting any correlation between contacts of any two pairs of chain fragments. The mean-field approximation is valid if $\lambda$-fragments discussed in Appendix $A$, are not swollen. Therefore for $\Omega \gtrsim 1$ this approximation is valid when the angle $\psi$ is large enough ( $\sin \psi \gtrsim \psi_{\mathrm{mf}}$, Eq. (A.5)). For $\Omega \lesssim 1$, the chains are never swollen on the scales corresponding to an anisotropic coil shape ( $L \lesssim l_{2}$ ), so that the mean field approximation is accurate for any angle $\psi$. However, a direct implementation of the mean field approximation to the problem of crumbly discs ordering is a delicate issue, as the correlation corrections to the mean field approximation could be significant for flat chain conformations, especially with regard to the angular dependencies of these corrections (see Sect. 4). We neglect here these corrections and discuss the background mean field approach.

As the volume fraction occupied by the links inside the "crumbly discs" is small, we can use the second virial approximation for the calculation of $U$. We introduce a length scale $\lambda$ such that

$$
d \ll \lambda \ll l \lesssim l_{2}
$$

and consider each disc as a solution of fragments of length $\lambda$ and diameter $d$. The concentration of these fragments inside the disc is $n_{\lambda} \equiv L /\left(\lambda R_{\|}^{2} R_{\perp}\right)$, and their second virial coefficient is [11]

$$
B_{\lambda}(\gamma) \simeq \lambda^{2} d(2 \sin \gamma+\pi d / \lambda)
$$

where $\gamma$ is the angle between the tangential (t) vectors of two fragments, which is assumed to be larger than $d / \lambda$, so that the second term in the r.h.s. of equation (B.2) can be neglected.

If two crumbly discs intersect at angle $\psi$, the interaction energy can be written as:

$$
\begin{gathered}
U \sim V_{\cap} n_{\lambda}^{2} B_{\lambda}, \quad \text { with } n_{\lambda} \sim L /\left(\lambda R_{\|}^{2} R_{\perp}\right), \\
B_{\lambda} \sim \lambda^{2} d b(\Psi), \quad b(\Psi)=2\langle\sin \gamma\rangle \simeq 1+\text { const. } \Psi^{2}, \quad \Psi=\sin \psi .
\end{gathered}
$$

Here $V_{\cap}$ is the typical volume of intersection of two discs defined in equation (A.1) (see Fig. 4a), $n_{\lambda}$ and $B_{\lambda}$ are the typical concentration and the second virial coefficient of $\lambda$-fragments, and the function $b(\psi)$ is used to describe the angular dependence of the average value of $\sin \left(\mathbf{t}, \mathbf{t}^{\prime}\right)$ for vectors $\mathbf{t}$ and $\mathbf{t}^{\prime}$ laying in the planes of the discs crossing each other at the angle $\psi$ (see Eq. (B.2) and Fig. 5). In the last equation (B.3) const. is a positive numerical factor of order unity, the particular value of this factor is irrelevant within the "scaling" approach; we thus put const. $=1$ for simplicity. Within the local mean-field approximation (Eq. (5.3)) the second virial coefficient of two chains is simply the sum of the excluded volumes due to all pairs of segments:

$$
B_{\mathrm{loc}}(\psi)=\left(\frac{L}{\lambda}\right)^{2} B_{\lambda}=L^{2} d b(\Psi) .
$$

For the most important region, $\psi \gtrsim R_{\perp} / R_{\|}$we get with equation (B.3):

$$
U \sim \frac{L^{2} d b(\Psi)}{R_{\|}^{3} \Psi}, \quad \Psi \equiv \sin \psi
$$

if the discs intersect; otherwise $U=0$. 


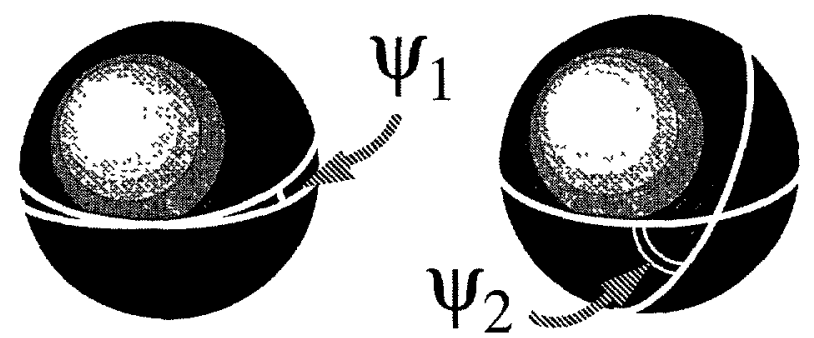

Fig. 5. - The sets of the main-chain orientations, $\mathbf{t}$ and $\mathbf{t}^{\prime}$ (shown as white lines) for two crumbly discs intersecting at the angle $\psi_{1}$ (left); $\psi_{2}$ (right). It is clear that $\left\langle\sin \left(\mathbf{t}, \mathbf{t}^{\prime}\right)\right\rangle$ is smaller for the left sketch as $\psi_{1}<\psi_{2}$.

\section{Appendix C}

\section{Validity of the Second Virial Approximation for Solutions of Crumbly (Loose) Discs}

In this Appendix we discuss the role of the higher order terms in the virial expansion.

The second virial approximation used in equations $(2.1,2.2,2.6)$ is accurate, if the corrections to the free energy functional (to the part $\mathcal{F}_{\text {int }}$ ) due to higher order terms of the virial expansion are small compared to the second virial contribution. We thus need to check whether the third virial term, $\mathcal{F}_{C}$, is smaller than second one, $\mathcal{F}_{\mathrm{B}}$ :

$\mathcal{F}_{\text {int }}=\mathcal{F}_{\mathrm{B}}+\mathcal{F}_{C}+\ldots$,

$\mathcal{F}_{\mathrm{B}} \sim c \int \beta\left(\mathbf{r}_{12}, \mathbf{n}_{1}, \mathbf{n}_{2}\right) f\left(\mathbf{n}_{1}\right) f\left(\mathbf{n}_{2}\right) \mathrm{d} \mathbf{r}_{12} \mathrm{~d} \Omega_{\mathbf{n}_{1}} \mathrm{~d} \Omega_{\mathbf{n}_{2}}$

$\mathcal{F}_{C} \sim c^{2} \iint \beta\left(\mathbf{r}_{12}, \mathbf{n}_{1}, \mathbf{n}_{2}\right) \beta\left(\mathbf{r}_{13}, \mathbf{n}_{1}, \mathbf{n}_{3}\right) \beta\left(\mathbf{r}_{32}, \mathbf{n}_{3}, \mathbf{n}_{2}\right) f\left(\mathbf{n}_{1}\right) f\left(\mathbf{n}_{2}\right) f\left(\mathbf{n}_{3}\right) \mathrm{d} \mathbf{r}_{12} \mathrm{~d} \mathbf{r}_{13} \mathrm{~d} \Omega_{\mathbf{n}_{1}} \mathrm{~d} \Omega_{\mathbf{n}_{2}} \mathrm{~d} \Omega_{\mathbf{n}_{3}}$

where $\beta\left(\mathbf{r}, \mathbf{n}_{1}, \mathbf{n}_{2}\right)=\beta^{\psi}(\mathbf{r})$ is the Mayer function, and $\psi$ is the angle between $\mathbf{n}_{1}$ and $\mathbf{n}_{2}$.

In the limit where the interaction is weak $\left(U_{\psi} \ll 1\right)$, a mean field consideration leads to

$$
\mathcal{F}_{\mathrm{B}} \sim c U_{\psi} V_{\psi}, \quad \mathcal{F}_{C} \sim c^{2} U_{\psi}^{3} V_{\psi}^{2}
$$

where the typical value of the Mayer's function is (see Eq. (B.5)):

$$
\beta^{\psi} \sim U_{\psi} \sim \frac{L^{2} d}{R_{\|}^{3} \sin \psi}
$$

provided that the discs intersect in space, i.e. that the vectors $\mathbf{r}_{\imath j}(i, j=1,2,3)$ in the integrals (C.1) belong to the characteristic volumes $V_{\psi}$ :

$$
V_{\psi} \sim \int \mathrm{d} \mathbf{r}_{\imath \jmath} \sim R_{\|}^{3} \sin \psi
$$

Thus, the condition that the second virial term dominates over the third one (i.e. that the second virial approximation is correct) for solutions of crumbly transparent discs $\left(U_{\psi} \lesssim 1\right.$, i.e $L<L_{6} \equiv l_{2} \Omega^{-2 / 5}$ ) is:

$$
c \ll \frac{1}{U_{\psi}^{2} R_{\|}^{3} \sin \psi} \sim \frac{R_{\|}^{3} \sin \psi}{\left(L^{2} d\right)^{2}},
$$


where $c$ is the chain concentration and $\sin \psi$ characterizes the typical orientation angle in the phase under consideration.

It turns out that for most of the "transparent disc" regimes the condition (C.5) is fulfilled. Indeed, one can easily check that with equation (2.9) the condition (C.5) can be written as $\psi \gg\left(L^{2} d\right)^{1 / 3} / R_{\|}=\left(\frac{L}{L_{6}}\right)^{1 / 6}$ Therefore the higher order terms in the virial expansion for transparent chains $\left(L<L_{6}\right)$ are not important near the $\mathrm{I} \rightarrow \mathrm{P}$ transition point where $\psi \sim 1$ (see Eq. (2.10)). The correlation corrections to the mean field approximation can however play a role in this region as shown in Section 4.

In the case where the discs are opaque $(U \gtrsim 1)$ a similar analysis shows that the third virial term is small when

$$
c \lesssim R_{\|}^{-3}
$$

In this case the disc-like coils are similar to ordinary rigid discs, and the Onsager approximation (see (2.2)) can be used for scaling estimates.

\section{References}

[1] Vogel T., Blatter K. and Schlüter A.-D., Makromol. Chem., Rapid Commun. 10 (1989) 427.

[2] Schlüter A.-D., Adv. Mater. 3 (1991) 282.

[3] Aggeli A., Boden N., Findlay J.B.C., Knowles P.F. and McLeish T.C.B., "Self-Assembly of Nanotapes based on Oligomeric Peptides", submitted to Nature.

[4] Nyrkova I.A., Semenov A.N., Joanny J.F. and Khokhlov A.R., J. Phys. II France 6 (1996) 1411.

[5] de Gennes P.G. and Prost J., The Physics of Liquid Crystals (Claredon Press, Oxford, 1993).

[6] Odijk T., Macromolecules 19 (1986) 2313.

[7] Semenov A.N. and Khokhlov A.R., Sov. Phys. Usp. 31 (1988) 988.

[8] Stroobants A., Lekkerkerker H.N.W. and Frenkel D., Phys. Rev. Lett. 57 (1986) 1452; Phys. Rev. A 36 (1987) 2929.

[9] Somoza A.M. and Tarazona P., Phys. Rev. Lett. 11 (1988) 2566.

[10] Taylor M.P., Hentschke R. and Herzfeld J., Phys. Rev. Lett. 62 (1989) 800, 1577; Phys. Rev. A 40 (1989) 1678.

[11] Onsager L., Ann. N. Y. Acad. Sci. 51 (1949) 627.

[12] Straley J.P., Mol. Cryst. Liq. Cryst. 22 (1973) 333; ibid 24 (1973) 7.

[13] Khokhlov A.R. and Semenov A.N., Physica A 108 (1981) 546.

[14] Khokhlov A.R. and Semenov A.N., J. Stat. Phys. 38 (1985) 161.

[15] Doi M. and Edwards S.F., The Theory of Polymer Dynamics (Academic Press, NY, 1986).

[16] Landau L.D. and Lifshitz E.M., "Statistical Physics", 3rd ed., Part 1, (Pergamon Press, Oxford, 1980).

[17] Grosberg A.Yu. and Khokhlov A.R., Statistical physics of macromolecules, Engl. transl. (AIP, NY, 1994).

[18] Schaefer D.W., Joanny J.F. and Pincus P., Macromolecules 13 (1980) 1280.

[19] Khokhlov A.R. and Semenov A.N., J. Phys. A 15 (1982) 1361.

[20] Nyrkova I.A., Semenov A.N. and Joanny J.F., J. Phys. II France 7 (1997) 847. 